\title{
Similarities Between Simulated Spatial Spectra of Scalp EEG, MEG and Structural MRI
}

\author{
Ceon Ramon · Walter J. Freeman • Mark Holmes • \\ A. Ishimaru · Jens Haueisen · Paul H. Schimpf · \\ Elham Rezvanian
}

Received: 29 December 2008 / Accepted: 4 June 2009/Published online: 26 June 2009

(C) The Author(s) 2009. This article is published with open access at Springerlink.com

\begin{abstract}
Electrical dipoles oriented perpendicular to the cortical surface are the primary source of the scalp EEGs and MEGs. Thus, in particular, gyri and sulci structures on the cortical surface have a definite possibility to influence the EEGs and MEGs. This was examined by comparing the spatial power spectral density (PSD) of the upper portion of the human cortex in MRI slices to that of simulated scalp EEGs and MEGs. The electrical activity was modeled with 2,650 dipolar sources oriented normal to the local cortical surface. The resulting scalp potentials were calculated with a finite element model of the head constructed from 51
\end{abstract}

Portions of these results were presented as a poster paper at the Biomag 2008, International Conference on Biomagnetism 2008, 25-29 August 2008, Sapporo, Japan; poster \#7-5.

C. Ramon $(\bowtie) \cdot$ A. Ishimaru

Department of Electrical Engineering, University of

Washington, Seattle, WA 98195, USA

e-mail: ceon@u.washington.edu; ceonramon@yahoo.com

C. Ramon

Department of Biomedical Engineering, Reykjavik University, Reykjavik, Iceland

W. J. Freeman

Department of Molecular and Cell Biology, University of California, Berkeley, CA 94720, USA

M. Holmes · E. Rezvanian

Department of Neurology, University of Washington, Seattle, WA 98195, USA

\section{J. Haueisen}

Institute of Biomedical Engineering and Informatics, Technical University Ilmenau, Ilmenau, Germany

\section{P. H. Schimpf}

Department of Computer Science, Eastern Washington

University, Cheney, WA 99004, USA segmented sagittal MR images. The PSD was computed after taking the fast Fourier transform of scalp potentials. The PSD of the cortical contour in each slice was also computed. The PSD was then averaged over all the slices. This was done for sagittal and coronal view both. The PSD of EEG and MEG showed two broad peaks, one from 0.05 to $0.22 \mathrm{cycles} / \mathrm{cm}$ (wavelength $20-4.545 \mathrm{~cm}$ ) and the other from 0.22 to 1.2 cycles $/ \mathrm{cm}$ (wavelength $4.545-0.834 \mathrm{~cm}$ ). The PSD of the cortex showed a broad peak from 0.08 to 0.32 cycles $/ \mathrm{cm}$ (wavelength $12.5-3.125 \mathrm{~cm}$ ) and other two peaks within the range of 0.32 to 0.9 cycles $/ \mathrm{cm}$ (wavelength $3.125-1.11 \mathrm{~cm}$ ). These peaks are definitely due to the gyri structures and associated larger patterns on the cortical surface. Smaller peaks in the range of 1-3 cycles/ $\mathrm{cm}$ were also observed which are possibly due to sulci structures. These results suggest that the spatial information was present in the EEG and MEG at the spatial frequencies of gyri. This also implies that the practical Nyquist frequency for sampling scalp EEGs should be $3.0 \mathrm{cycles} / \mathrm{cm}$ and an optimal interelectrode spacing of about $3 \mathrm{~mm}$ is needed for extraction of cortical patterns from scalp EEGs in humans.

Keywords EEG spectra - EEG simulations . Cortex spectra - Head models - Brain models . Brain surface $\cdot$ Gyri $\cdot$ Sulci $\cdot$ FEM $\cdot$ PSD

\section{Introduction}

The human cortical surface is marked by gyral folds separated by sulcal walls with the gyral crowns averaging $1-3 \mathrm{~cm}$ in width and length. Gyrification contributes to the amplitudes of electroencephalographic fields over the scalp. It is due to differing distances of scalp sites from 
cortical surfaces and also due to orientations of cortical current dipoles with respect to the scalp surface (Freeman et al. 2003). Gyral crowns and more superficial aspects of the sulcal walls give rise to higher EEG and MEG amplitudes while deeper aspects of the sulcal walls that are farther from the EEG electrodes and MEG sensors give lower amplitude recordable signals. In addition, the high skull impedance with low impedances in the scalp above and the cerebrospinal fluid below acts as a low pass filter that smooths the EEG differences. This spatial low-pass filter effect of the CSF/bone/skin is much smaller on MEG as compared to its effect on EEG. However, MEG sensors are typically at larger distances to the sources and thus will have smaller effects on spatial frequencies of MEG data sets. In general, EEG electrodes are about $16 \pm 3.9 \mathrm{~mm}$ and MEG sensors are about $48 \pm 5.7 \mathrm{~mm}$ from the pial surface (Freeman et al. 2009).

The question arises, what is the spatial resolution of the EEG? Specifically, do the spatial frequencies of the cortical surface imposed by gyri and sulci cause peaks in the spatial power spectral density (PSD) of multichannel simulated EEG and MEG? This we examined with a highly heterogeneous finite element method (FEM) model of an adult male subject. The model was used to simulate the scalp EEG and MEG from the sources confined to the gray matter in the upper portion of the cerebral hemispheres. The spatial power spectral densities in the surface dimensions $\left(\mathrm{PSD}_{x}\right.$ and $\left.\mathrm{PSD}_{z}\right)$ of the EEG, MEG and cortical contours were computed and compared in log-log coordinates in order to display the spectral ranges of greatest interest. Our results show that the spatial broad peaks due to gyri structures were present in the cortical spectra and also in the spatial spectra of the EEG and MEG. These peaks had a good correlation. More details of these are given in the "Results" and "Discussion" sections. These findings suggest that the conformance of the electrical dipoles to the cortical contours does influence the spatial spectra of EEG and MEG. A further implication is that for maximal retrieval of information from measurement and display of the textures of spatial patterns in scalp EEGs and MEGs will require Nyquist sampling at spatial wavelengths less than the dimensions of gyri by using arrays with interelectrode spacings of approximately $3 \mathrm{~mm}$.

\section{Methods}

For computation of scalp EEGs and MEGs, a finite element method (FEM) model of the head was used. Our model building details have been described earlier (Haueisen et al. 2002; Ramon et al. 2004, 2006a, 2006b). For the sake of completeness, a summary is provided here. The T1 weighted sagittal MRI slices of an adult male subject with
$3.2 \mathrm{~mm}$ thickness were collected with a 1.5 Tesla GE Signa scanner. The original MR slices were of $256 \times 256$ resolution with $1.0 \mathrm{~mm}$ size pixels (Haueisen et al. 2002; Ramon et al. 2006a, 2006b). A total of 51 contiguous slices was used. The MR images were segmented using a semiautomatic tissue classification program developed by us. The identified tissues were: scalp, fat, muscle, hard skull bone, soft skull bone, gray matter, white matter, eyes, spinal cord and cerebellum, cerebrospinal fluid (CSF) and soft tissue. A detailed structure of the eye sockets, sinus and oral cavities, and occipital hole was also included in the segmentation. The segmented images were sub-sampled to a $2 \times 2 \mathrm{~mm}$ resolution for computational work. One of the segmented slices, marked as slice number 30 is shown in Fig. 1. This is the 30th slice starting from the left side of the subject. It is $1.7 \mathrm{~cm}$ to the right from the midline of the brain. The $x$ coordinate increases from anterior (front) to the posterior (back) of the subject, the $y$ coordinate increases from superior (top of the head) to the inferior (bottom) and the $z$ coordinate increases from left to the right side of the subject.

The FEM model had a voxel resolution of $2 \times$ $2 \times 3.2 \mathrm{~mm}$. There were 835,584 hexahedral voxels and 865,332 nodes for the model. The tissue resistivity values used in the model were compiled from published values (Geddes and Baker 1967; Foster and Schwan 1989; Gabriel

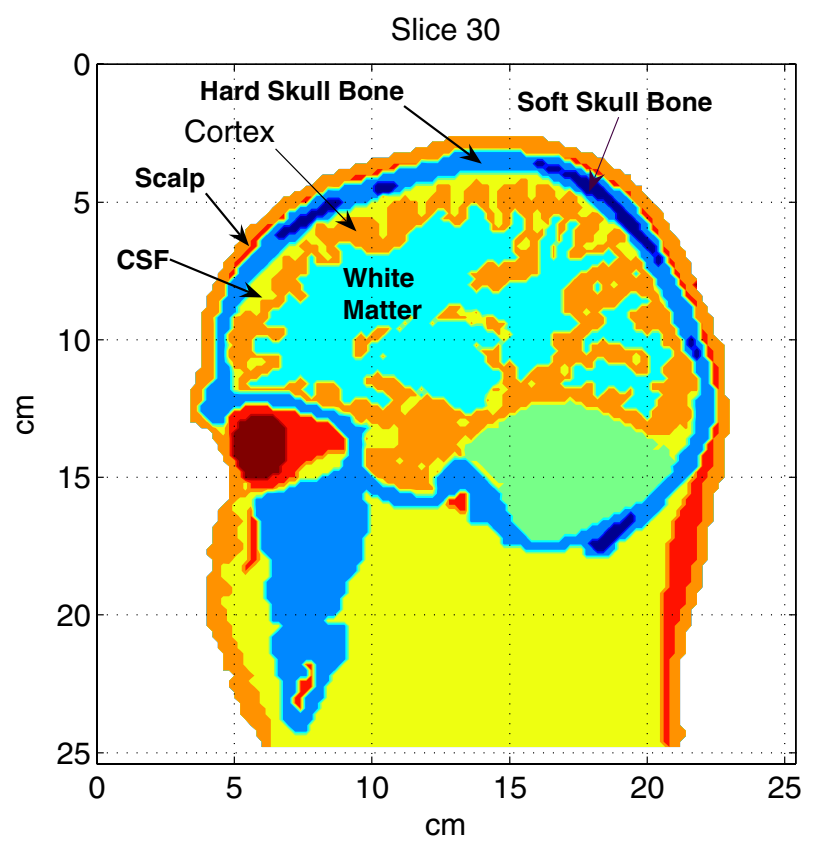

Fig. 1 A segmented image slice with major tissues identified in it. The cortical contour formed by the boundary of CSF and the gray matter was used for dipole locations to model the electrical activity. The same contour was used for computing the PSD of the cortical contours. Only the top portion of the contour, above $9 \mathrm{~cm}$, was used for modeling and analysis work 
et al. 1996). These complied tissue resistivity values have been used by us before and are listed in our previous papers (Ramon et al. 2004, 2006a, 2006b). Using a uniform finite element solver (Schimpf et al. 1998), the scalp potentials were computed due to dipolar sources confined to the gray matter in the upper portion of the cerebral hemispheres. This covered all the cortical voxels from the middle of the forehead to the top of the head. As an example, in the slice 30 shown in Fig. 1, the used cortical voxels were between 4.5 and $9 \mathrm{~cm}$ on the vertical scale. This choice was made because the dipolar sources in the upper portion of the cerebral hemispheres contributed most to the scalp EEGs and MEGs as compared to the sources located in the lower, or deeper, portions of the cerebral hemispheres. A total of 2,650 dipoles were used. The dipoles were oriented normal to the local cortical surface. The dipoles were placed at the voxel nodes which were in the middle of the gray matter layer. The orientation and surface normals were computed using the surface defined by the adjacent grid points near to the dipole. The dipole moments were random with a uniform distribution in the range of $0.0-0.1 \mathrm{~mA}$-meter. The electrical flux densities and potentials in the whole head model were computed. The scalp potentials and magnetic fields were extracted from the computed FEM model results. The magnetic coils were assumed to be located $1.0 \mathrm{~cm}$ above the scalp on a curved surface. The scalp potentials in each slice at the pixel vertices with $2 \mathrm{~mm}$ resolution were extracted from the computed FEM model results. For plotting and PSD computations of the EEG and MEG data, we used a flat surface of $15 \times 15 \mathrm{~cm}$ with a gird resolution of $2 \times 3.2 \mathrm{~mm}$. This will refer to $2 \mathrm{~mm}$ resolution in anterior to posterior direction and $3.2 \mathrm{~mm}$ resolution in left to right direction. The size of the flat surface was large enough to cover the top portion of the head above the forehead level.

We performed 100 trial runs with different random dipole moments. The PSD of EEG and MEG for each run was computed and then averaged over all the trials. The spatial profiles of scalp potentials were different for each run. However, their spatial PSD profiles were only slightly different from one run to the next. This is because the spatial locations and the orientation of the dipoles are fixed and only their intensities are randomly changing. This will be evident in the errors bars of the PSD of scalp EEGs and MEGs averaged over 100 trials. Refer to Figs. 2 and 3 in the "Results" section. The error bars in the averaged spatial PSD plots were not significantly different from one trial to the next when averaged over more than 20 runs. Thus, averaging over 100 runs is more than adequate for the results presented here. The PSD was computed after taking the fast Fourier transform (FFT) of the 2-D plot of the scalp EEG or MEG data.

The PSD of the upper portion of cortical contour in sagittal and coronal slices was also computed. In each slice, the cortical contour defined by the boundary of the gray matter and the CSF was extracted. In the slice 30 shown in Fig. 1, it will be the boundary of CSF and gray matter between 4.5 and $9 \mathrm{~cm}$ on the vertical scale. The contour was flattened and its FFT was taken. After that the spatial PSD was computed. The $x$ component of the PSD, called $\mathrm{PSD}_{x}$, was computed in sagittal slices and averaged over all the slices. The $z$ component of the PSD, called $\mathrm{PSD}_{z}$, was computed in coronal slices and averaged over all the slices.

All computations were performed on an Intel $2.4 \mathrm{GHz}$, quad-core workstation with 8.0 gigabytes of memory. Postprocessing, PSD computations and visualizations were
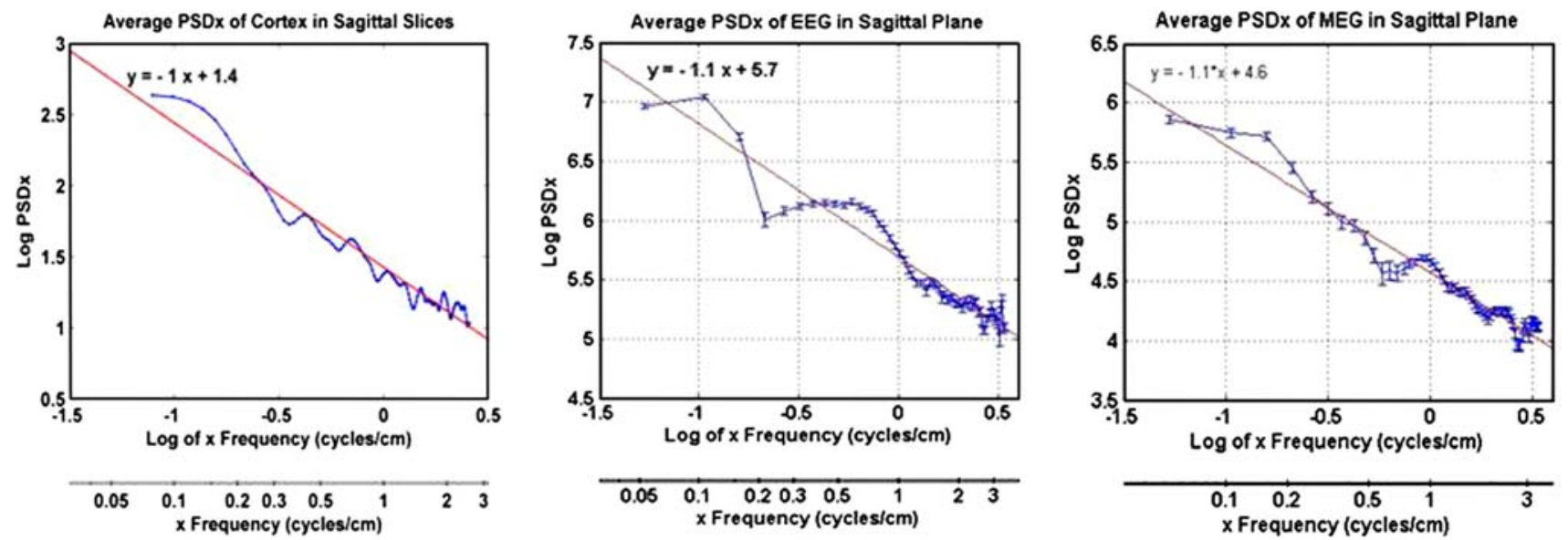

Fig. 2 Averaged $\mathrm{PSD}_{x}$ of cortex, EEG and MEG in sagittal slices. Large broad peaks at mid frequencies $(0.3-1.0 \mathrm{cycles} / \mathrm{cm})$ are possibly due to gyral structures 

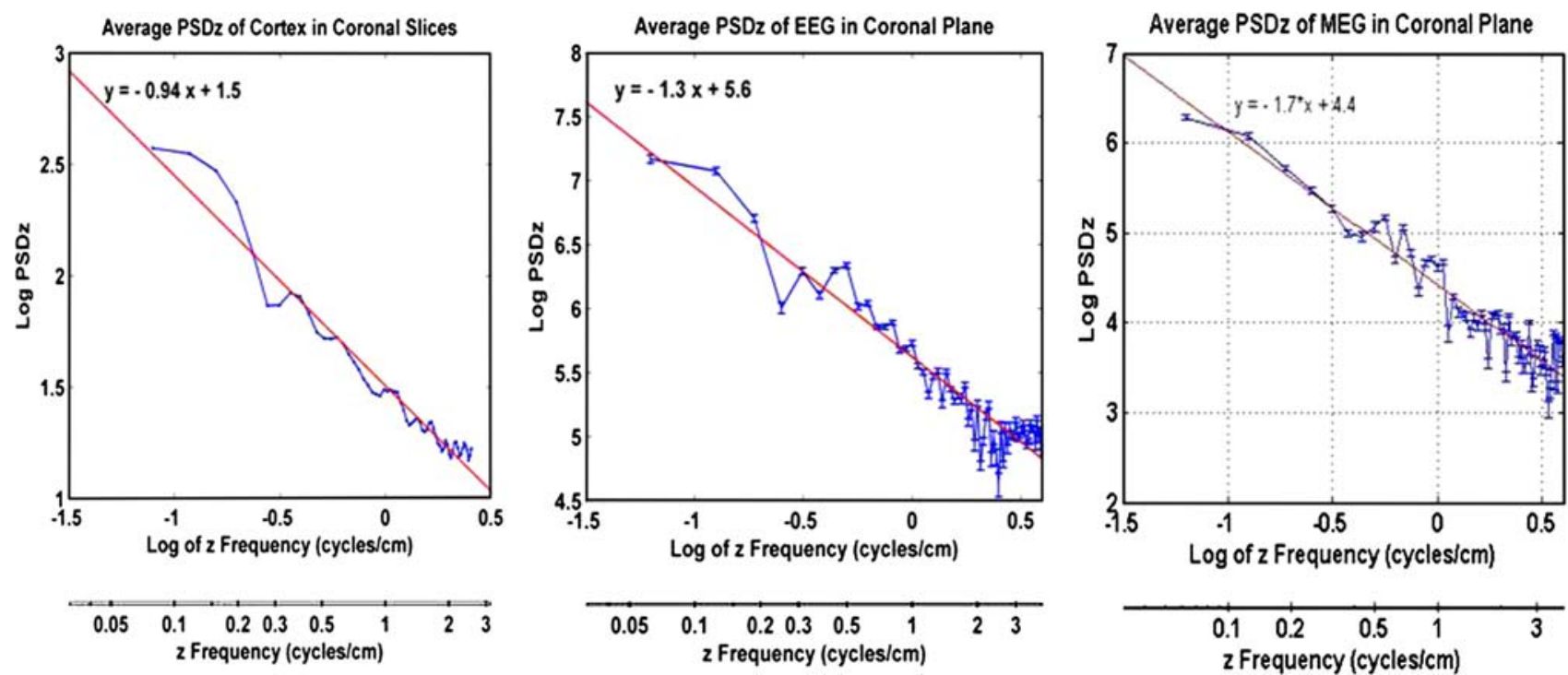

Fig. 3 Averaged $\mathrm{PSD}_{z}$ of cortex, EEG and MEG in coronal slices. Large broad peaks at mid frequencies $(0.3-1.0 \mathrm{cycles} / \mathrm{cm})$ are possibly due to gyral structures

done with the Matlab software, version 7.1 (Mathworks, Inc., Natick, MA).

\section{Results}

The averaged $\mathrm{PSD}_{x}$ of the cortex, scalp potentials and magnetic fields in sagittal slices are given in Fig. 2. Similarly, the averaged $\mathrm{PSD}_{z}$ of the cortex, scalp potentials and magnetic fields in coronal slices are given in Fig. 3. The sagittal plane contains the $x$ frequencies while the coronal plane contains the $z$ frequencies. The plots are for the log of PSD versus $\log$ of frequency values. A linear frequency scale is given at the bottom of the figure.

In Fig. 2 for sagittal slices, a low frequency broad peak from 0.08 to 0.32 cycles $/ \mathrm{cm}$ (wavelength $12.5-3.125 \mathrm{~cm}$ ) is predominant in the cortical spectra and it is very similar to the low frequency $(0.05-0.22$ cycles $/ \mathrm{cm}$; wavelength $20-4.545 \mathrm{~cm}$ ) broad peak in the $\mathrm{PSD}_{x}$ of EEG and MEG. This broad peak is due to the whole cortical surface, left and right lobes and other larger structures on the cortical surface. Following this broad peak, there are two distinct peaks in the cortical $\mathrm{PSD}_{x}$. One is from 0.32 to 0.65 cycles/ $\mathrm{cm}$ (wavelength $3.125-1.54 \mathrm{~cm}$ ) and the other is from 0.65 to 0.9 cycles $/ \mathrm{cm}$ (wavelength $1.54-1.11 \mathrm{~cm}$ ). These are due to gyral structures on the cortical surface. There is another peak from 0.9 to 1.3 cycles/cm (wavelength 1.11$0.77 \mathrm{~cm}$ ) which possibly could be related to sulci structures. Beyond this, other peaks are difficult to distinguish from the noise. $\mathrm{PSD}_{x}$ of EEG has a broad peak from 0.3 to 1.2 cycles $/ \mathrm{cm}$ (wavelength $3.34-0.834 \mathrm{~cm}$ ) while MEG has one prominent peak from 0.6 to 1.5 cycles/cm (wavelength $1.67-0.67 \mathrm{~cm}$ ).
In Fig. 3 for the coronal plane, there are two prominent broad peaks for the $\mathrm{PSD}_{z}$. One is from 0.05 to 0.22 cycles/ $\mathrm{cm}$ (wavelength $20-4.54 \mathrm{~cm}$ ) and the other is from 0.22 to 1.2 cycles $/ \mathrm{cm}$ (wavelength $4.545-0.834 \mathrm{~cm}$ ). The second peak $(0.22-1.2$ cycles $/ \mathrm{cm})$ can be further broken down to smaller peaks, particularly, for the coronal slices (left plot in Fig. 3). The first peak is influenced by the electrical activity of dipoles on the large contour of the cortical surface which extends from 7 to $19.6 \mathrm{~cm}$ on the horizontal scale as shown in Fig. 1. The second peak, 0.221.2 cycles $/ \mathrm{cm}$, reflects the gyri structures on the cortical surface. There are some noticeable peak structures in the $2.0-3.0$ cycles $/ \mathrm{cm}$ (wavelength $0.5-0.34 \mathrm{~cm}$ ) range. These are possibly due to the electrical activity of dipoles embedded in sulci structures of $3-5 \mathrm{~mm}$ width in the cortex.

The $\mathrm{PSD}_{x}$ of EEG and MEG as shown in Fig. 2 has a linear slope of -1.1 and the $\operatorname{PSD}_{x}$ of the cortex has a linear slope of -1.0 . These values are very close to each other. In comparison, from Fig. 3 the linear slopes of the $\mathrm{PSD}_{z}$ of EEG is -1.3 , of MEG is -1.7 and that of the cortex is -0.94 . These values are not very close to each other. The measured time-domain spectrum of human scalp EEGs also has a linear slope which is very close to -1.0 (Freeman et al. 2003).

\section{Discussion}

The similarity of the anatomical and functional spectra indicates that the spatial information was present in the scalp EEG and MEG at the spatial frequencies of gyri. The gyri frequencies, 0.3-1 cycles/cm (wavelength 3.34- 
$1.0 \mathrm{~cm}$ ), are readily observable in a broad peak in the PSD spectrum of scalp EEG and MEG. In contrast the PSD of cortical contours show a predominant low frequency broad peak from 0.08 to 0.24 cycles $/ \mathrm{cm}$ (wavelength $12.5-$ $4.167 \mathrm{~cm}$ ) which relates to the whole cortical surface and to the half lobes. The smaller peaks in 0.32 to 1.0 cycles/ $\mathrm{cm}$ (wavelength $3.125-1.0 \mathrm{~cm}$ ) range are related to the gyral structures on the cortical surface.

The electrical dipoles which produce the scalp potentials are located within the cortex and their locations correctly follow the gyri and sulci geometry. However, the volume currents emanating out of the dipoles traverse through the CSF, skull bone and other tissues before reaching the scalp. The skull bone influences the scalp potentials (Ramon et al. 2004) and acts as a lowpass filter in temporal domain (Pfurtscheller and Cooper 1975) and also in spatial domain (Cooper et al. 1965). In the spatial domain it was found that cortical recordings with electrodes separated by just few millimeters had different spectral characteristics, while this was not true for scalp recordings (Cooper et al. 1965). This spatial lowpass filtering effect of the skull bone could be one of the reasons that we see more features in the PSD of cortical contours as compared with that of scalp EEGs. The CSF also plays a role in spreading the volume currents which affects the scalp potentials (Ramon et al. 2004, 2006a). This could also influence the power spectral densities of scalp EEGs. This has not been studied before and should be examined.

The broad peak at low frequencies $(<0.24$ cycles $/ \mathrm{cm})$ observed in cortical PSD and also in the PSD of scalp EEG and MEG is definitely due to the top portion of the cortex taken as a whole. As an example, as shown in Fig. 1, the top portion of the cortical contour used here extended from 7 to $19.6 \mathrm{~cm}$ on the horizontal scale. This provides the lowest spatial frequency of $(1 /(19.6-7.0))=0.08$ cycles/ $\mathrm{cm}$. A combination of many gyri on the cortical contour could give rise to several other frequencies in the range of $0.08-0.3$ cycles/cm (wavelength $12.5-3.34 \mathrm{~cm}$ ). Beyond that, individual gyri of width $1-3 \mathrm{~cm}$ will give rise to peaks in the $0.33-1.0$ cycles $/ \mathrm{cm}$. Thus, the observed broad low frequency peak in the averaged PSD of the cortex and that of scalp EEG and MEG is definitely due to the upper cortical surface of the brain.

There are some other peaks above the $1.0 \mathrm{cycle} / \mathrm{cm}$ mark. These are possibly due to sulci structures in the cortex. Refer to Figs. 2 and 3. These peaks are difficult to ascertain due to the background computational noise in the averaged PSD plots of EEG and MEG. Average sulci width of $3.4 \mathrm{~mm}$ has been suggested earlier based on the CT scans of 44 normal adults (Gyldensted and Kosteljanetz 1975). However, the width of sulci could range from 1.0 to $8 \mathrm{~mm}$ in a normal adult. This is very easy to see in MR images of the brain with a $1.0 \mathrm{~mm}$ resolution. Thus, peaks in the range of $1-3$ cycles/cm could be attributed to the sulci structures.

A possibility of an artifact should also be examined. Our slice thickness was $3.2 \mathrm{~mm}$ which will give rise to a frequency of 3.12 cycles $/ \mathrm{cm}$. Thus, spatial frequency variations above 3.12 cycles/cm are not meaningful due to the limited resolution of the FEM model. However, peaks less than 3.12 cycles $/ \mathrm{cm}$ should be considered to be free from computational noise and FEM model based errors.

In summary, we do see the anatomical spectra of gyri superimposed on the spatial spectra of scalp EEGs and also on MEGs. Sulci spatial frequencies are visible in the spectra of cortical contours, but are difficult to ascertain in the spectra of scalp EEGs and MEGs. A better model of the head with a resolution of $1.0 \mathrm{~mm}$ will be needed to examine the effects of sulci on the scalp EEGs and MEGs.

The 1/f form of linear fit shown in Figs. 2 and 3 is for the spatial domain. In time-domain, The PSD of EEG, in general, has a slope of unity but deviations from unity have also been observed. Time-domain power in excess of $1 / \mathrm{f}$ is often present in the gamma $(30-100 \mathrm{~Hz})$ range (Freeman et al. 2003). Spatial spectra need not conform to the $1 / \mathrm{f}$ dependence as observed in the time-domain PSD plots. The structure of gyri and sulci will influence the texture of the PSD in spatial domain which one could observe in the 2-D surface plots of PSD on the scalp surface. The slopes will give a general indication of the similarities or differences between two PSD plots. In Fig. 2 for sagittal slices, the slopes of the $\mathrm{PSD}_{x}$ of EEG, MEG and cortex are very close to each other while they are different in Fig. 3. This would suggest that the spatial plots of EEG, MEG and cortical contours will have more similarities in sagittal planes as compared with coronal planes. However, this needs to be further investigated with a higher resolution $(\sim 1 \mathrm{~mm})$ head model.

A prominent question to ask will be: what should be the interelectrode spacing to extract the gyri information from the scalp EEG or MEG data? Looking at the Figs. 2 and 3, the highest gyri related frequency will be about 1.5 cycles/ $\mathrm{cm}$. This implies that the practical Nyquist frequency for sampling scalp EEG should be about 3.0 cycles $/ \mathrm{cm}$. This will give us an optimal interelectrode spacing of about $3.3 \mathrm{~mm}$ for human scalp EEGs. Thus an interelectrode spacing of less than $3.3 \mathrm{~mm}$, such as, $3 \mathrm{~mm}$ may be a good choice. A one dimensional curvilinear electrode array made using gold-plated connector pins threaded into a band of embroidery fabric with interstices at $3 \mathrm{~mm}$ intervals has been used before for scalp EEG recordings (Freeman et al. 2003). It is feasible to extend this to a 2-D grid electrode arrangements for small areas of scalp. A $16 \times 16$ electrode grid with $3 \mathrm{~mm}$ spacing might be feasible to build.

We have used best available values of tissue conductivity values for EEG and MEG simulations (Ramon et al. 
2006a, 2006b). These values match well with the recently measured and estimated skull and brain tissue conductivities (Lai et al. 2005; Oostendorp et al. 2000). The observed effects will be slightly different if the subject-specific tissue conductivity values are used. The anisotropic conductivities of gray and white matter also influence the EEG and MEG simulations (Haueisen et al. 2002; Wolters et al. 2006). It needs to be examined how the tissue anisotropies influence the PSD of the scalp EEGs and its correlation with cortical PSD.

Open Access This article is distributed under the terms of the Creative Commons Attribution Noncommercial License which permits any noncommercial use, distribution, and reproduction in any medium, provided the original author(s) and source are credited.

\section{References}

Cooper R, Winter AL, Crow HJ, Walter WG (1965) Comparison of subcortical, cortical and scalp activity using chronically indwelling electrodes in man. Electroencephalogr Clin Neurophysiol 18:217-228

Foster KR, Schwan HP (1989) Dielectric properties of tissues and biological material: a critical review. Crit Rev Biomed Eng 17:25-104

Freeman WJ, Burke BC, Holmes MD, Vanhatalo S (2003) Spatial spectra of scalp EEG and EMG from awake humans. Clin Neurophysiol 114:1053-1068

Freeman WJ, Ahlfors SP, Menon V (2009) Combining fMRI with EEG and MEG in order to relate patterns of brain activity to cognition. Int J Psychophysiol 73:43-52

Gabriel S, Lau RW, Gabriel C (1996) The dielectric properties of biological tissues: III. Parametric models for the dielectric spectrum of tissues. Phys Med Biol 41:2271-2293
Geddes LA, Baker LE (1967) The specific resistance of biological material-a compendium of data for the biomedical engineer and physiologist. Med Biol Eng 5:271-293

Gyldensted C, Kosteljanetz M (1975) Measurements of the normal hemispheric sulci with computer tomography: a preliminary study on 44 adults. Neuroradiology 10:147-149

Haueisen J, Tuch DS, Ramon C, Schimpf P, Wedeen VJ, George JS, Belliveau JW (2002) The influence of brain anisotropy on human EEG and MEG. Neuroimage 15:159-166

Lai Y, van Drongelen W, Ding L, Hecox KE, Towle VL, Frim DM, $\mathrm{He}$ B (2005) Estimation of in vivo human brain to skull conductivity ratio from simultaneous extra and intracranial electrical potential recordings. Clin Neurophysiol 116:456-465

Oostendorp TF, Delbeke J, Stegeman DF (2000) The conductivity of the human skull: results of in vivo and in vitro measurements. IEEE Trans Biomed Eng 47:1487-1492

Pfurtscheller G, Cooper R (1975) Frequency dependence of the transmission of the EEG from cortex to scalp. Electroencephalogr Clin Neurophysiol 38:93-96

Ramon C, Schimpf P, Haueisen J, Holmes M, Ishimaru A (2004) Role of soft bone, CSF and gray matter in EEG simulations. Brain Topogr 16:245-248

Ramon C, Schimpf P, Haueisen J (2006a) Influence of head models on EEG simulations and inverse source localizations. Biomed Eng Online 5:10

Ramon C, Haueisen J, Schimpf PH (2006b) Influence of head models on neuromagnetic fields and inverse source localizations. Biomed Eng Online 5:55

Schimpf P, Haueisen J, Ramon C, Nowak H (1998) Realistic computer modeling of electric and magnetic fields of human head and torso. Parallel Comput 24:1433-1460

Wolters CH, Anwander A, Tricoche X, Weinstein D, Koch MA, MacLeod RS (2006) Influence of tissue conductivity anisotropy on EEG/MEG field and return current computation in a realistic head model: a simulation and visualization study using highresolution finite element modeling. Neuroimage 30:813-826 